\title{
Erratum
}

\section{The Kashiwara conjugation and wave-front sets of regular holonomic distributions on a complex manifold}

\section{Emmanuel Andronikof}

Invent. math. 111, 35-49 (1993)

Corrections are indicated between quotation marks "()"

p. 37 line 7

" $d \geqq 1$ " (instead of $d>1$ ).

p. 39 line 10

" $U \subset T^{*} X "\left(\right.$ instead of $\left.U \subset{ }^{*} X\right)$

p. 39 line 11

the conclusion of the proposition should be:

"Then supp $u$ contains any irreducible component of Car $\mathscr{M}$ which it meets at a smooth point of Car $\mathscr{M}$."

In fact, as was pointed out to us by Motoo Uchida, the given proof only shows that supp $u$ propagates along the smooth part of Car $\mathscr{M}$. However, this does not affect the other results.

\section{p. 42 lines $6,7,8$}

The last sentence should be replaced by the following one:

"Put $S=\operatorname{supp} s p(u)$. From the previous equality $\mathbb{C}^{\times} S=\Lambda$ it follows, on one hand, that $S$ meets all the irreducible components of $A$, hence $S \cap \Lambda_{\text {reg }}=\Lambda_{\text {reg }}$ by proposition 2.3; on the other hand, $\overline{S \cap \overline{A_{\text {reg }}}}=S$, whence $A=S=W F_{A}(u)$." 